\title{
Progress and challenges in understanding core transport in tokamaks in support to ITER operations
}

\author{
P Mantica1, C Angioni'2, N Bonanomi' ${ }^{2}$, Citrin ${ }^{3}$, B A Grierson 4 , \\ F.Koechl ${ }^{5}$, A Mariani ${ }^{1}$, G M Staebler ${ }^{6}$, Eurofusion JET ${ }^{7}$ and MST18 \\ contributors, ASDEX Upgrade team ${ }^{9}$ and ITPA transport $\&$ con- \\ finement group ${ }^{10}$
}

\footnotetext{
${ }^{1}$ Istituto per la Scienza e Tecnologia dei Plasmi, Consiglio Nazionale delle Ricerche, Milano, Italy

${ }^{2}$ Max-Planck-Institut für Plasmaphysik, D-85748 Garching, Germany

${ }^{3}$ DIFFER - Dutch Institute for Fundamental Energy Research, Eindhoven, The Netherlands

${ }^{4}$ Princeton Plasma Physics Laboratory, Princeton University, Princeton, NJ 08543, USA

${ }^{5}$ UKAEA Culham Centre for Fusion Energy, Abingdon, Oxon, OX14 3DB, UK

${ }^{6}$ General Atomics, San Diego, CA 92186-5608, USA
}

E-mail: paola.mantica@,cnr.it

\begin{abstract}
Fusion performance in tokamaks depends on the core and edge regions as well as on their nonlinear feedbacks. The achievable degree of edge confinement under the constraints of power handling in presence of a metallic wall is still an open question. Therefore, any improvement in the core temperature and density peaking is crucial for achieving target performance. This has motivated further progress in understanding core turbulent transport mechanisms, to help scenario development in present devices and improve predictive tools for ITER operations. In the last two decades, detailed experiments and their interpretation via the gyrokinetic theory of turbulent transport have led to a satisfactory level of understanding of the heat, particle, and momentum transport channels and of their mutual interactions. This paper presents some highlights of the progress, which stems from joint work of several devices and theory groups,

in Europe and worldwide within the ITPA (International Tokamak Physics Activities) framework. On the other hand, the achievement of predictive capabilities of plasma profiles via integrated modeling, which also accounts for the nonlinear interactions inherent to the multi-channel nature of transport, is a priority in view of ITER. This requires using faster, reduced models, and the extent to which they capture the complex physics described by nonlinear gyrokinetics must be carefully evaluated. Present quasi-linear models match well experiments in baseline scenarios, and thus offer reliable predictions for the ITER reference scenario, but have issues in advanced scenarios. Some of these challenges are examined and discussed. In the longer term, advances in high performance computing will continue to drive physics discovery through increasingly complex gyrokinetic simulations, allowing also further development of reduced models. The development of neural network surrogate models is another recent advance that bridges the gap towards physics-based fast models for optimisation and control applications.
\end{abstract}

Keywords: core turbulent transport, critical gradient, stiffness, multi-scale, isotope scaling, model validation

\footnotetext{
${ }^{7}$ See the author list of "E. Joffrin et al. Nuclear Fusion in press (https://doi.org/10.1088/1741-4326/ab2276)"

${ }^{8}$ See author list of "B. Labit et.al. 2019 Nucl. Fusion in press (https://doi.org/10.1088/1741-4326/ab2211)"

${ }^{9}$ See author list of "H. Meyer et.al. 2019 Nucl. Fusion in press (https://doi.org/10.1088/1741-4326/ab18b8)"

${ }^{10} \mathrm{See}$ https://portal.iter.org/departments/POP/ITPA/TC/Pages/default.aspx
} 


\section{Introduction}

In the quest for thermonuclear fusion power in tokamak devices, the improvement of energy confinement is a key objective. Global confinement in $\mathrm{H}$-mode scenarios ( $\mathrm{H}$ stands for High confinement due to the presence of an edge transport barrier commonly called "pedestal") depends on both pedestal and core profiles. In presence of a metallic wall -required to avoid Tritium retention- the achievable degree of edge confinement under the constraints of power handling is still an open question. Therefore, any improvement in the peaking of ion temperature and density -within scenario tolerance- is crucial for achieving target performance, since fusion power goes as the square of the central ion pressure. This has motivated a vigorous effort during the last two decades, to understand and eventually control the turbulent processes that determine radial transport of heat, particles and momentum in the tokamak core, overcoming in most situations the wellassessed collisional neoclassical transport mechanisms. Substantial progress has been achieved, thanks to the development of "physics comprehensive" nonlinear gyrokinetic (GK) models and simulations and the design of sophisticated transport experiments and advanced diagnostics. By "physics comprehensive" we mean that the non-linear GK model includes all main physics ingredients that are required to provide a realistic description of the type of phenomenology under consideration, namely the experimental observations of core transport in mostly MHD quiescent plasmas. The computational cost of GK simulations is however such that predictions of plasma profiles and their time evolution is not affordable. Therefore, "reduced" first principle models have been developed, with the aim of providing viable tools in support to the operations of present and future devices. Reduced models then have to be able to also include those ingredients that allow approximating the results of the nonlinear GK codes in the range of parameters where they are used. These models have provided good reproduction of $\mathrm{H}$-mode baseline scenarios - fully inductive scenarios with standard confinement with respect to $\mathrm{H}$-mode scaling and moderate $\beta$ (ratio of thermal to magnetic pressure) - and therefore can now be used for scenario optimization in present machines and for physics based predictions of future devices. Going beyond the baseline, to partially inductive or fully noninductive advanced scenarios, both featuring improved confinement with respect to the baseline and high $\beta$, or moving from Deuterium plasmas to different gases, the present reduced models are seen to show some limitations. A continuous process of improvement in the way reduced models capture the complex physics described by nonlinear GKs is therefore ongoing, and represents the final challenge for providing reliable predictive tools for the core plasma profiles in ITER. A few examples of recent experimental results that have further confirmed the high level of accuracy of GK simulations and have indicated paths for improvement of reduced models will be discussed.

\section{Turbulent transport}

In the tokamak configuration, the helicoidal magnetic field (B) lines lie on a set of nested toroidal flux surfaces. Since transport $\| \mathbf{B}$ is much faster than $\perp \mathbf{B}$, temperature and density (in the limit of low centrifugal effects) are constant on a flux surface and transport of heat, particles and momentum is in the radial direction. Transport can be described by a set of fluid equations, derived from the Vlasov equation (allowing for a suitable collision operator) by taking moments of the distribution function [1]. For density and temperature these are:

$$
\begin{aligned}
& \frac{\partial n_{e}}{\partial t}+\boldsymbol{\nabla} \cdot \boldsymbol{\Gamma}_{e}=S_{e 0} \\
& \frac{3}{2} \frac{\partial\left(n_{e, i} T_{e, i}\right)}{\partial t}+\boldsymbol{\nabla} \cdot\left(\boldsymbol{q}_{e, i}+\frac{5}{2} T_{e, i} \boldsymbol{\Gamma}_{e, i}\right)=S_{e, i}
\end{aligned}
$$

where $n_{e}$ is the electron density, $T_{e, i}$ the electron, ion temperature, $\Gamma_{\mathrm{e}}$ the particle flux, $\mathbf{q}_{\mathrm{e}, \mathrm{i}}$ the ion, electron heat flux, $\mathrm{S}_{\mathrm{e} 0}$ the particle source and $\mathrm{S}_{\mathrm{e}, \mathrm{i}}$ the electron, ion heat sources. Similar equations can be written for impurity and toroidal momentum transport.

In a tokamak the radial fluxes are in most conditions carried dominantly by turbulent fluctuations [2,3] in electrostatic (ES) potential $\phi, \mathrm{n}, \mathrm{T}$ and $\mathbf{B}$, which are characterized by a broadband spectrum around the diamagnetic frequency, by a small relative fluctuation level, and by $k_{\|} \ll k_{\perp} \sim 1 / \rho$ ( $\rho$ is the Larmor radius $\rho=(m T)^{1 / 2} / q B$ with $m$ and $\mathrm{q}$ the particle mass and charge). A $3 \mathrm{D}$ visualization of turbulence in a tokamak from a simulation by the GK code GYRO [4] is shown in Figure 1.

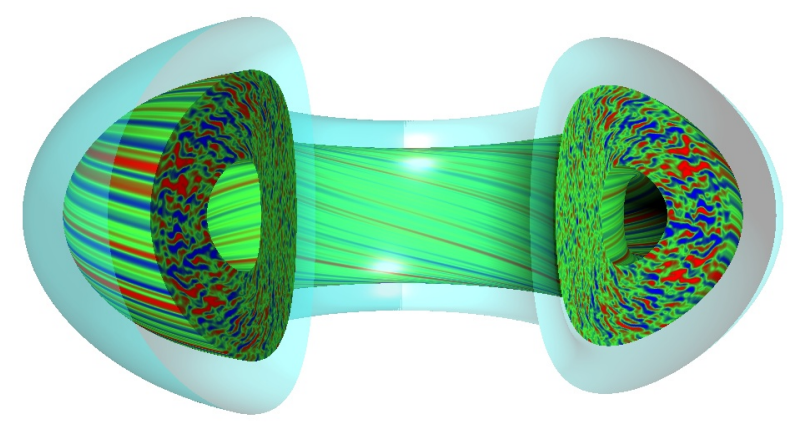

Figure 1. 3D visualizations of contour plots of ES potential fluctuations from a simulation by GYRO. From [5].

The radial fluxes in the case of ES turbulence are due to the fluctuations of the radial component of the ExB velocity, $\widetilde{v_{E}}$

$$
\boldsymbol{\Gamma}_{\boldsymbol{e}}=\left\langle\widetilde{n_{e}} \widetilde{\boldsymbol{v}_{\boldsymbol{E}}} \cdot \boldsymbol{e}_{\boldsymbol{r}}\right\rangle \quad \boldsymbol{q}_{\boldsymbol{e}, \boldsymbol{i}}=\frac{3}{2} n\left\langle\widetilde{T_{e, l}} \widetilde{\boldsymbol{v}_{\boldsymbol{E}}} \cdot \boldsymbol{e}_{\boldsymbol{r}}\right\rangle
$$

where $<>$ denotes time and flux surface averages and $\mathbf{e}_{\mathrm{r}}$ the radial vector.

Defining temperature and density inverse scale-lengths as

$$
\frac{R}{L_{T}}=-\frac{R \nabla T}{T} \quad \text { and } \quad \frac{R}{L_{n}}=-\frac{R \nabla n}{n}
$$

with $\mathrm{R}$ the tokamak major radius, linear instabilities are excited above a critical value of $\mathrm{R} / \mathrm{L}_{\mathrm{T}}$, which we will call "threshold", as sketched in Figure 2a. 
The main instabilities on the scale of the ion Larmor radius are the Ion Temperature Gradient (ITG) modes, driven by $\mathrm{R} / \mathrm{L}_{\mathrm{Ti}}$, and the Trapped Electron Modes (TEM), driven by $\mathrm{R} / \mathrm{L}_{\mathrm{Te}}$ and $\mathrm{R} / \mathrm{L}_{\mathrm{n}}$. On the scale of the electron Larmor radius we have the Electron Temperature Gradient (ETG) modes, driven by $\mathrm{R} / \mathrm{L}_{\mathrm{Te}}[6]$.

Non-linear saturation of these modes occurs via interaction across different scales. In particular, axisymmetric Zonal Flows regulate transport by shearing drift wave structures and facilitating energy damping $[2,3]$.

A strongly non-linear response of turbulence to the driving temperature gradient impacts the peaking of the temperature profile, as shown in Figure 2, where the heat flux is typically normalized to gyro-Bohm (GB) units [6]:

$q_{\text {norm }}=q /\left(n T^{2} \rho_{s}\right) /\left(e B R^{2}\right)$ with $\rho_{s}=\left(m_{i} T\right)^{1 / 2} / e B$

A basic theoretical description of turbulent diffusion as a random walk process with spatial step the ion Larmor radius and time step the inverse of the diamagnetic frequency leads to the expectation that the heat diffusivity scales as $\chi \sim \frac{T}{e B} \rho_{*} F\left(v_{*}, \beta, \ldots\right)$, with $\rho_{*}$ and $v_{*}$ the normalized ion Larmor radius and collisionality [7]. This has been named gyroBohm scaling, hence the choice of the GB normalization of fluxes. Defining "stiffness" as $\partial \mathrm{q}_{\text {norm }} / \partial\left(\mathrm{R} / \mathrm{L}_{\mathrm{T}}\right)$, we see that for high stiffness $\mathrm{R} / \mathrm{L}_{\mathrm{T}}$ cannot depart significantly from the threshold, irrespective of power, and particularly at reactorrelevant high temperatures (due to the $1 / \mathrm{T}^{5 / 2}$ dependence of the GB normalized flux). This implies that the core $T$ can only be increased by enhancing the pedestal, which may create issues for plasma wall interaction. Therefore, one target of transport research has been to understand how to increase threshold or decrease stiffness, to achieve high central $\mathrm{T}$ avoiding the need of a high pedestal.
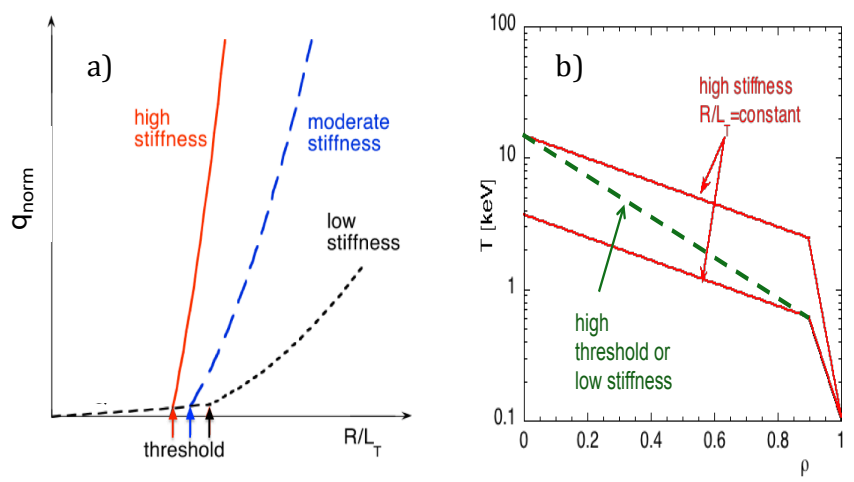

Figure 2. a) Sketch of the turbulent heat flux response to the driving gradient $R / L_{T}$. b) Sketch of the effect of stiffness on the temperature profile.

\section{Experimental techniques for transport studies}

Experiments specifically aimed at determining critical gradient and stiffness can be made by two techniques: 1) heat flux scan (Figure 3) [8], consisting in varying the repartition of power between two sources at different radial positions, in order to change the temperature gradient at constant edge temperature, and reconstruct the flux-gradient relation (Figure 4$)$; 2) power modulation [9], to measure the heat wave propagation, which is sensitive to the incremental diffusivity $\chi^{i n c}=-\partial q / n \partial \nabla T$ and therefore provides an independent determination of the stiffness (Figure 4). These experiments provide far more constraining tests for validating theoretical models than a pure match of steady-state profiles or the power balance diffusivity $\chi^{P B}=-q / n \nabla T$.

In addition, a powerful validation method for turbulence codes is the comparison with fluctuation measurements, with more and more advanced diagnostics becoming available. Fluctuation amplitudes, frequency spectra, radial correlation lengths and phase angles between e.g. $\mathrm{n}$ and $\mathrm{T}$ have been measured and compared with theoretical predictions in AUG, C-MOD, DIII-D, TCV. Progress on this has been recently reviewed in [10].

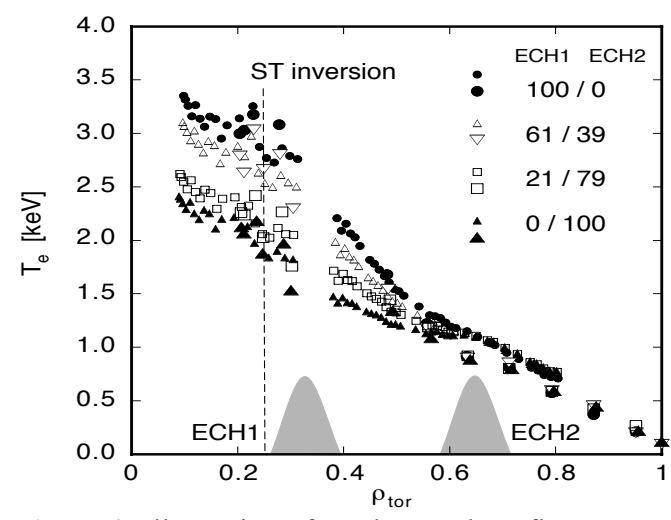

Figure 3. Illustration of an electron heat flux scan experiment on AUG. From [8].

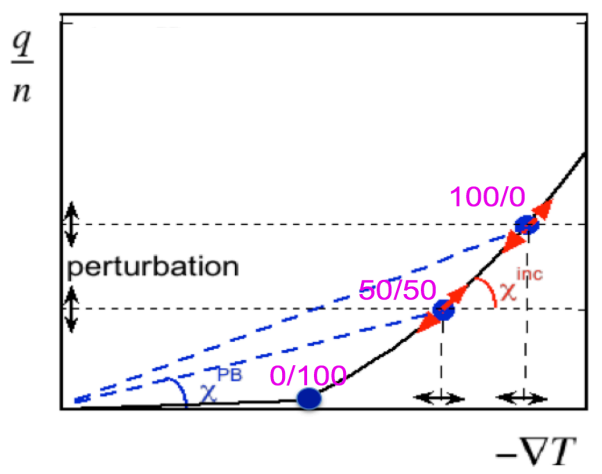

Figure 4. Sketch of the reconstruction of the $q(\nabla T)$ curve from heat flux scan and power modulation experiments such as those shown in Figure 3. The fractions of on- and off-axis power are indicated. The figure refers to a radial position in between onand off-axis depositions.

\section{Non-linear gyrokinetic and reduced models}

Non-linear GK codes (see e.g. [11,12] for general reviews) have been developed to provide a physics comprehensive description of turbulent transport. They solve the 5D GK equation (derived from the $6 \mathrm{D}$ collisional Vlasov equation removing the gyro-angle dependence) coupled with the Maxwell equations. In this paper we focus on local $\delta$ codes, which solve for a small perturbation of the distribution function $\delta \mathrm{f} / \mathrm{f} \sim \rho / \mathrm{R}<<1$ in a flux tube spatial domain, illustrated in Figure 5, with periodic boundary conditions. Results will be quoted from the codes GENE [13], GKW [14], GYRO [4]. The local $\delta$ approximation still provides a realistic descrip- 
tion of core turbulence in most experimental conditions whilst allowing pragmatic simulation and systematic comparison with experiments. This is supported by extensive verification and validation work, e.g. in [15-22] regarding heat transport, which is the main focus of this paper, but also regarding main particle, impurity and momentum transport (see e.g. [23-28]). On the basis of such extensive work, we can now state that local of GK theory is an adequate description to explain most (not all) experimental observations related to core turbulence in present medium-sized and large tokamaks. Some recent examples will be illustrated in detail in Sect.6. Gyrokinetic codes are computationally very expensive, ranging from 1-100 CPUh for a linear run, to $10^{4}-10^{6}$ CPUh for a single scale non-linear run, to $>10^{7} \mathrm{CPUh}$ for a multi- scale non-linear run. Therefore, they cannot be used for simulating full radial profiles of plasma parameters, nor their time evolution, as required for experiment interpretation or in predictive mode to assist plasma scenario optimization.

Reduced, faster models have been developed using a quasilinear approximation, i.e. computing fluxes as $F^{Q L}=C \sum_{k_{y}} w_{k_{y}}^{Q L} F_{k_{y}}^{\text {Lnorm }}$

where $\mathrm{k}_{\mathrm{y}}$ is the wave number with respect to the $\mathrm{y}$ direction, $\mathrm{C}$ is a scalar factor calibrated against non-linear GK simulations, the $\mathrm{F}_{\mathrm{ky}}{ }^{\text {Lnorm }}$ are normalized fluxes calculated from linear eigenmodes. The choice of weighting factors constitutes the saturation rule of the quasi-linear model, approximating the nonlinear physics that sets the saturation amplitude and flux spectrum as observed in nonlinear simulations. $w_{k y}^{Q L}$ are functions of the linear growth rate $\gamma_{\mathrm{k}}$ and the perpendicular wave number $k_{\perp}$. A common choice is for example [29, 36] $w_{k_{y}}^{Q L} \sim\left(\frac{\gamma_{k_{y}}}{\left\langle k_{\perp}^{2}\right\rangle}\right)^{\xi}$

where $<>$ indicates the flux-surface average and $\xi$ is an adjustable exponent to be tuned for best match of the non-linear spectra in a given range of parameters.

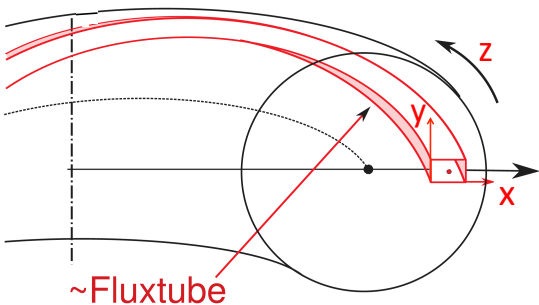

Figure 5. Sketch of the field-aligned coordinates used in turbulent transport models. $(\mathrm{x}, \mathrm{y}, \mathrm{z})$ represent the radial, the binormal and the parallel positions respectively $(\mathrm{x}=$ const and $\mathrm{y}=$ const define a magnetic field line, while $\mathrm{z}$ sets the position along that line).

In this paper, we will consider results from the two most recently developed quasi-linear models: TGLF [30,31], which is a gyrofluid electromagnetic (EM) transport model using shaped geometry, and QuaLiKiz [32-35], which is a GK ES transport model using shifted-circle geometry. TGLF features two versions of the saturation rule: SAT0 [30], which is local in $\mathrm{k}_{\mathrm{y}}$, and SAT1 [31], which features a non-linear upshift of the critical threshold and multi-scale interactions. Extensive verification and validation has been carried out for these models, e.g. in [36-44], and some recent results will be discussed in sects.5 and 6 .

The advantage of quasi-linear models is that they are fast $(\sim$ seconds for a single call) and therefore can be integrated into a transport code, which solves the transport equations using the quasi-linear models to calculate the turbulent fluxes, in order to compute the full profile time evolution.

As a final remark we note that, in all comparisons with experiment of GK simulations or quasi-linear modeling, the collisional neoclassical transport is always taken into account on top of the turbulent one, using well established codes like NCLASS [45] or NEO [46]. The neoclassical component is typically significant for ion heat transport in the very central region and for heavy impurities, particularly in presence of poloidal asymmetries.

\section{Integrated modeling}

With the term "integrated modeling" we refer to plasma scenario simulations in which many different aspects of a tokamak discharge are modeled self-consistently. In this paper, the term will indicate simulations of the core part of the plasma, taking the top of the pedestal as a boundary condition, integrating the aspects of MHD equilibrium, power, particle and momentum sources, and $\alpha$-particle heating in case of D-T plasmas, and transport of different channels $\left(n_{e}\right.$ or $n_{i}, T_{e}, T_{i}$, rotation, impurity density). This type of modeling allows the study of the nonlinear multi-channel interactions and is used for scenario modeling, prediction and optimization. Achieving routine use in integrated modeling is the primary goal of the quasi-linear model development, since at the moment this is not possible with non-linear GK codes, due to their computational cost.

A taste of the success of integrated modeling of baseline scenarios is provided in Figures 6 and 7. Figure 6 [47] shows a 3-field $\left(\mathrm{n}_{\mathrm{e}}, \mathrm{T}_{\mathrm{e}}, \mathrm{T}_{\mathrm{i}}\right)$ simulation of a 1.74 T/1.3 MA low-torque DIII-D ITER baseline scenario, with both $3.3 \mathrm{MW}$ of Electron Cyclotron Heating $(\mathrm{ECH})$ and 2.6 MW of Neutral Beam Injection (NBI) or only 3.5 MW NBI. The simulation has been made with the TGYRO stationary-state transport solver [48] and the TGLF SAT0 model. Good agreement with experimental profiles is evident.
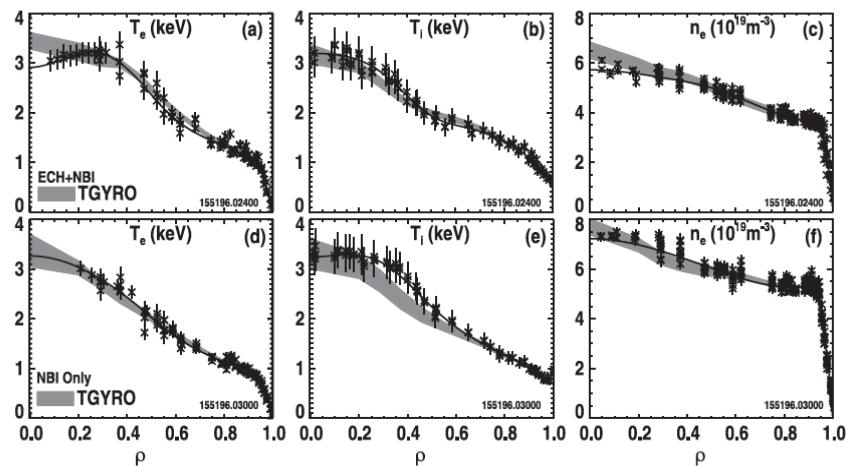

Figure 6. Simulations of low-torque DIII-D ITER baseline profiles by TGYRO with TGLF SAT0. Case with ECH+NBI heating (upper row) and NBI only (bottom row). From [47].

Figure 7 shows a 4-field $\left(\mathrm{n}_{\mathrm{e}}, \mathrm{T}_{\mathrm{e}}, \mathrm{T}_{\mathrm{i}}\right.$, toroidal rotation) simulation of a JET 2.7 T/ 3MA high power $\mathrm{H}$-mode plasma with $28 \mathrm{MW}$ of NBI and $5 \mathrm{MW}$ of Ion Cyclotron Heating (ICRH). 
The simulation of rotation allows a self-consistent prediction of the ExB flow shear stabilization of turbulent transport, which is an example of nonlinear multi-channel interaction. The simulation has been made with the JINTRAC transport code suite [49] and the QuaLiKiz model. Also in this case a very good agreement is obtained.
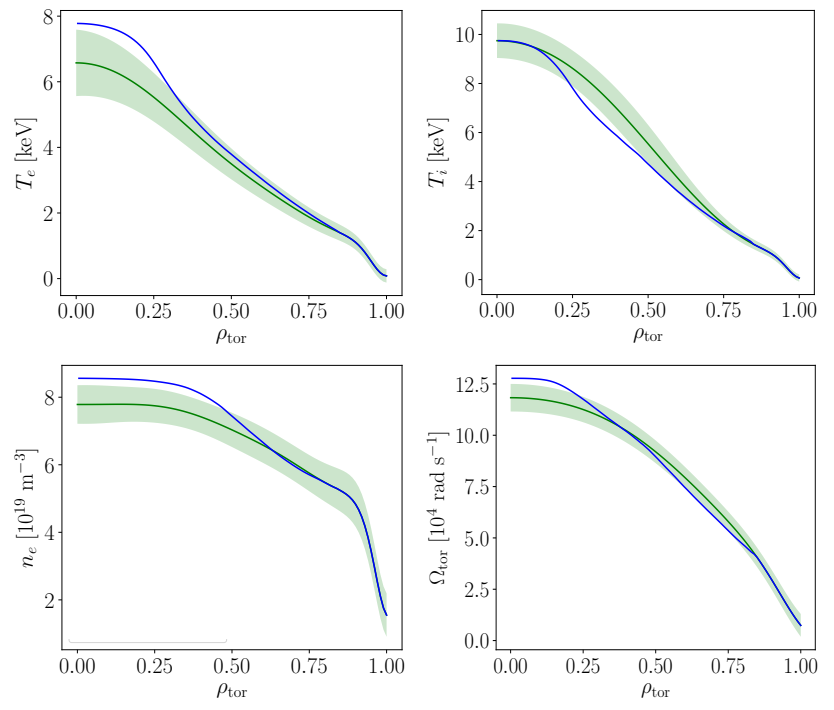

Figure 7. Simulation of JET high power baseline discharge 92436 at $\mathrm{t}=10 \mathrm{~s}$ using JINTRAC with QuaLiKiz. The blue line is the simulation, the green line and bands are the experimental profile fits and their $1 \sigma$ uncertainty obtained by a Gaussian Process Regression method. From [50].

The success of the two quasi-linear models in describing baseline scenarios in present machines increases our confidence in physics-based predictions for the core of ITER baseline plasmas. Figure 8 shows simulations using JINTRAC+QuaLiKiz and TGYRO+TGLF-SAT0/SAT1 for the ITER D-T (50\% mixture) fully inductive reference scenario at 5.3 T/15 MA, with $33 \mathrm{MW}$ NBI power and $20 \mathrm{MW}$ ECH power located at $\rho_{\text {tor }}=0.4$ ( $\rho_{\text {tor }}$ is the square root of the normalized toroidal magnetic flux). The details of the JINTRAC simulation are reported in [51]. In particular, EPED [52] has been used to set the pedestal pressure to $\sim 130 \mathrm{kPa}$, close to the ideal MHD limit. All 3 simulations have the same heating and particle source profiles, as well as ${ }^{4} \mathrm{He}$ boundary condition at $1 \%$. The average fusion power prediction is $\mathrm{P}_{\text {fus }} \sim 510$ $\mathrm{MW}, \mathrm{Q}_{\text {fus }}=\mathrm{P}_{\text {fus }} / \mathrm{P}_{\text {ext }} \sim 9.5\left(\mathrm{P}_{\text {ext }}\right.$ is the external heating), with a thermal confinement time $\tau_{\mathrm{E}}=\mathrm{W}_{\text {th }} / \mathrm{P}_{\text {tot }}$ of $2.35 \mathrm{~s}\left(\mathrm{~W}_{\text {th }}\right.$ is the total thermal energy and $P_{\text {tot }}$ the total heating, including $\alpha$ power and not subtracting radiated power). The agreement amongst the models is reasonable. The GB normalized flux is rather low in the ITER core, due to the high temperature, implying that the threshold prediction has more impact on the profiles than the stiffness prediction. Still, capturing threshold and particle transport physics is not trivial and the agreement of the two models is highly encouraging, indicating that the approximations to GK equations in QuaLiKiz and the gyrofluid closures in TGLF seem justified to capture the physics needed in this parameter regime. It is interesting to note that this confinement time is similar to that predicted by the IPB98 $(y, 2)$ 0D scaling law [53] for these discharge parameters $(\sim 2.53 \mathrm{~s})$. These physics based simulations then support the possibility of reaching $\mathrm{Q}_{\text {fus }}$ values in the right ball park with respect to the original $\mathrm{Q}_{\text {fus }}=10$ target. It is worth noting that this prediction is dependent also on the pedestal assumptions, whose discussion goes beyond the scope of this paper [54]. What is important is that now we have validated physics based models for the core transport that will be used for further scenario optimization accounting also for core-edge coupling [51].

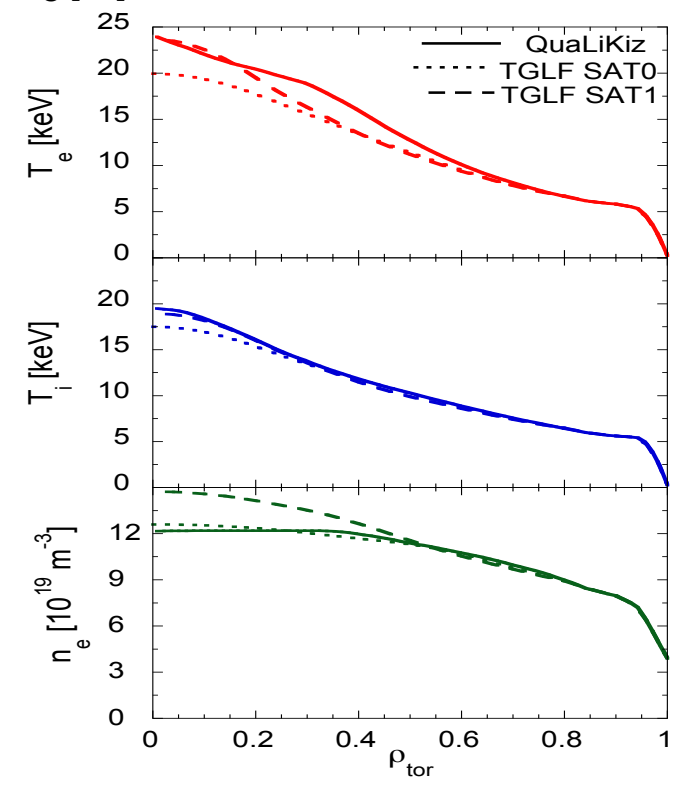

Figure 8. Simulations of ITER reference baseline profiles by TGYRO with TGLF SAT0/SAT1 and by JINTRAC with QuaLiKiz.

The ITER Research Plan [55] is not limited to the baseline scenario, but features operation in advanced scenarios, which target reduced $\mathrm{Q}_{\text {fus }}$ but extended burning time at lower plasma current and increased non-inductive current fraction, with improved confinement and high $\beta$. It is therefore important to evaluate on present machines the performance of quasi-linear models in reproducing this kind of scenarios. Unfortunately, the reliability of quasi-linear models in advanced scenarios is not as good as for baseline scenarios. This is primarily due to the increased importance of EM effects in advanced scenarios, as will be discussed in subsequent sections. In addition, (narrow) internal transport barrier formation remains a challenge for local GK models in general. This exposes the need for understanding the governing physics with nonlinear GKs, and improving quasi-linear transport models for integrated modeling of these scenarios.

Figure 9 shows the 4-field JINTRAC+QuaLiKiz simulation of the JET $2 \mathrm{~T} / 1.7 \mathrm{MA}$ high power advanced discharge 75225, with $17 \mathrm{MW}$ NBI power. The magenta lines indicate a significant underestimate of $\mathrm{T}_{\mathrm{i}}, \mathrm{n}_{\mathrm{e}}$ and rotation peaking in the inner half-radius. TGLF apparently performs better in JET advanced scenarios, as documented in [56]. However, subsequent tests of the ion stiffness level of the model indicated a generally too low ion stiffness level in TGLF [44, 57-59] -see also Figure 15- which suggests great care in the application of TGLF to these regimes, in which the ion stiffness level is shown by GK simulations to be very important, as will be discussed in sect.6.1. 

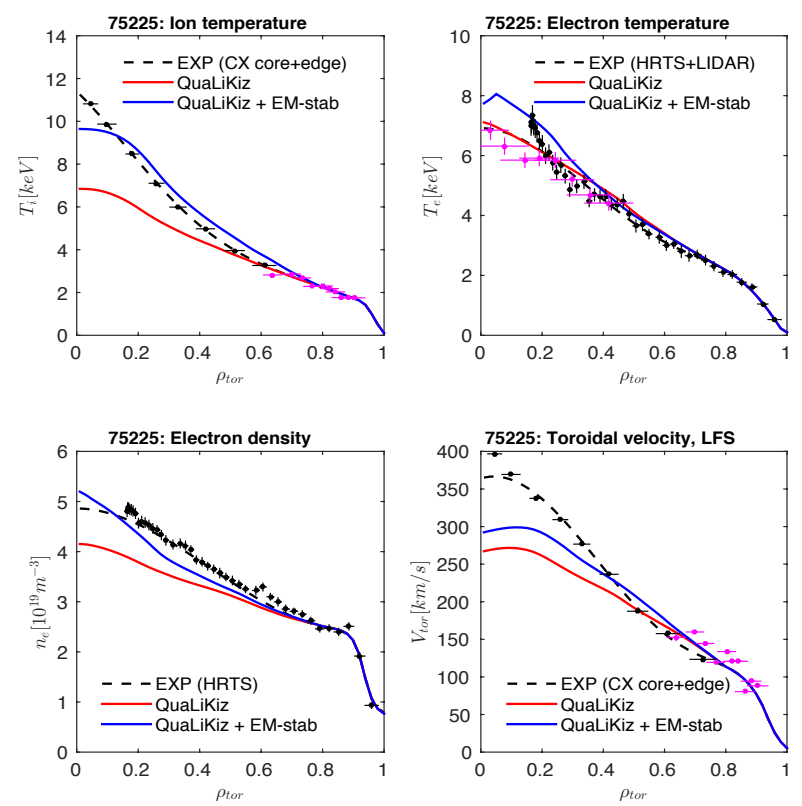

Figure 9. Simulations of JET advanced discharge 75225 by JINTRAC with QuaLiKiz. Two cases are reported, without (magenta) and with (blue) the ad hoc correction for ITG EM stabilization discussed in Sect.6.1. From [35, 60].

A continuous process of improvement of quasi-linear models is therefore ongoing, to enrich their physics content and expand their range of validity. This process is generally constituted by two phases: 1) the interpretation of new experimental results is sought using the gyro-kinetic theory until good understanding of the physics mechanisms is reached; 2) the quasi-linear model is then compared, to judge if it is adequately matching experiment and GK theory, otherwise the missing effects need to be identified and included in the quasilinear model. In Sect.6 three recent examples of new experimental results and theoretical findings that have raised new challenges for existing quasi-linear models will be discussed, in the spirit of stimulating further work towards improved predictive tools for ITER operations beyond the baseline scenarios.

\section{Recent progress in understanding and new challenges for improvement of quasi-linear models}

\subsection{The stabilizing effects of fast ions}

An ion heat flux scan using ICRH in $\left({ }^{3} \mathrm{He}\right)-\mathrm{D}$ minority scheme in JET L-mode plasmas at different values of NBI power showed a significant reduction of ion stiffness at high power (Figure 10a), leading to $T_{i}$ profiles very peaked in the central region (Figure 10b) [61,62]. Very similar increase of central $T_{i}$ peaking was obtained in AUG in $\mathrm{H}$-mode plasmas adding ICRH in $\left({ }^{3} \mathrm{He}\right)-\mathrm{D}$ minority to NBI [63]. An ion heat flux scan using on- and off-axis NBI on AUG evidenced a significant decrease in ion stiffness in the cases with on-axis NBI [64].
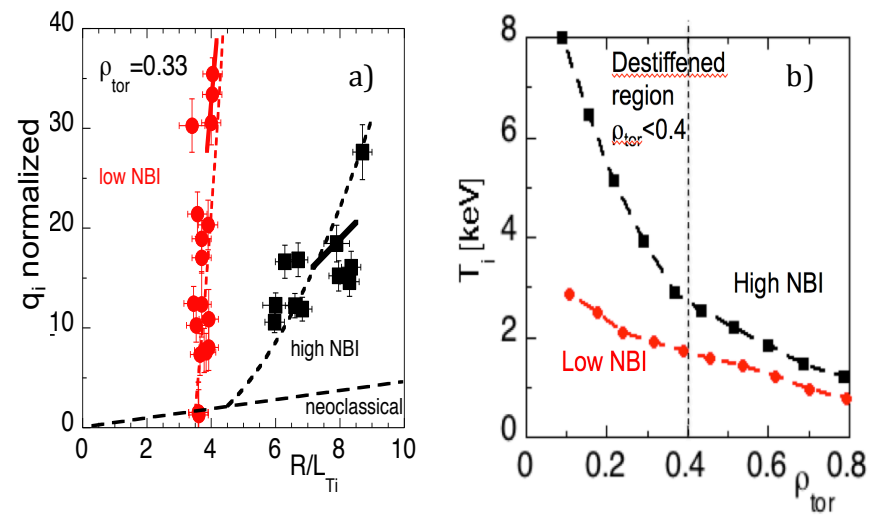

Figure 10. a) Ion heat flux scan in a JET L-mode plasma at low and high NBI power. b) $T_{i}$ profiles for two JET L-mode shots at low and high NBI power at the upper end of the heat flux scan. From [61].

The JET result was reproduced well in non-linear GENE gyro-kinetic simulations and found due to a significant stabilization of ITG turbulence by fast ions from NBI and ICRH [65], as illustrated in Figure 11. These findings were later successfully benchmarked against GYRO simulations [17], whilst GKW simulations of the AUG heat flux scan also evidenced the role of NBI fast ions in the ion stiffness reduction [64]. The physics mechanisms behind the stabilization have been investigated in detail with GENE for the JET case. Part of the stabilization is due to an ES resonant linear effect associated to the ICRH fast ions [66], and part is due to a nonlinear EM effect caused by non-linear coupling of ITG, marginally stable Toroidal Alfven Eigenmodes and Zonal Flows [67]. This second mechanism is analogous to the non-linear EM stabilization of ITGs by the thermal plasma $\beta$ (i.e. the ratio of thermal to magnetic pressure) due to interaction between unstable modes, stable modes, and zonal flows, described in [68]. Such thermal $\beta$ stabilization adds to the two fast ion effects in high $\beta$ scenarios (see e.g. results in JET and AUG in $[69,70])$, whilst for the low $\beta$ L-mode shot of Figure 11 the stabilization is mainly due to the fast ion mechanisms. It is important to assess if the fast ion effects will be important also in ITER, in addition to the thermal $\beta$ non-linear EM stabilization. The ES linear mechanism will vanish for high energy $\alpha$-particles, but some effects in relation to ICRH fast ions may be present. Likely however the fast ion nonlinear EM effect will be more important, including both NBI and ICRH fast ions and $\alpha$ particles. An estimate of the impact of fast ions in an ITER D-T advanced scenario using GENE is reported in [71], with a significant reduction of ion stiffness when adding NBI and $\alpha$ fast ions ( $\alpha$ pressure being 3 times the NBI one), as shown in Figure 12. Unfortunately, these non-linear EM effects (both from fast ions and from thermal $\beta$ ) are not included in the presently available quasilinear models, leading to an over-estimate of ion heat transport in those scenarios where ion stiffness plays a role, like the L-mode or the advanced scenarios. This is in fact the reason for the issues found in the QuaLiKiz simulation of the JET advanced discharge in Figure 9. Proper physics based modifications to the saturation rule to account for such effects have yet to be found, and work in this direction is ongoing. In the mean time, an ad hoc correction has been introduced in QuaLiKiz, by rescaling $\mathrm{R} / \mathrm{L}_{\mathrm{Ti}}$ by a factor $\beta_{\text {thermal }} / \beta_{\text {total }}[35]$, which also implicitly assumes that the thermal EM- 
stabilisation (linked with total $\beta$ ) is correlated with suprathermal pressure due to high NBI and ICRH heating. This correction was found to reproduce reasonably a number of JET advanced scenario simulations, including the case in Figure 9 (blue curve).

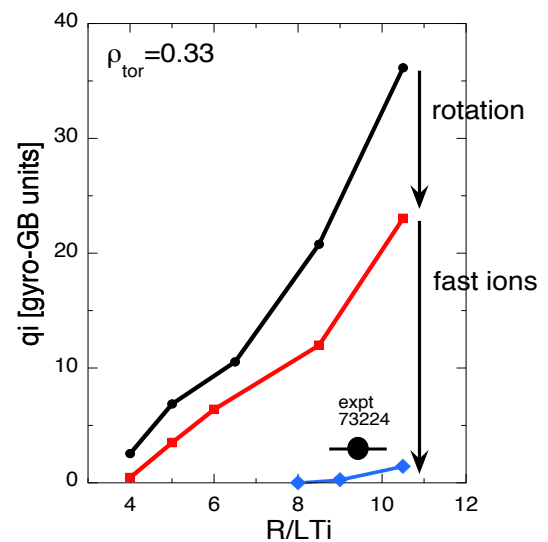

Figure 11. GENE non-linear simulations of the JET high NBI case (shot 73224) of Figure $10 \mathrm{~b}$ at $\rho_{\text {tor }}=0.33$, showing the stabilizing effect of rotation and fast ions. Adapted from [65].

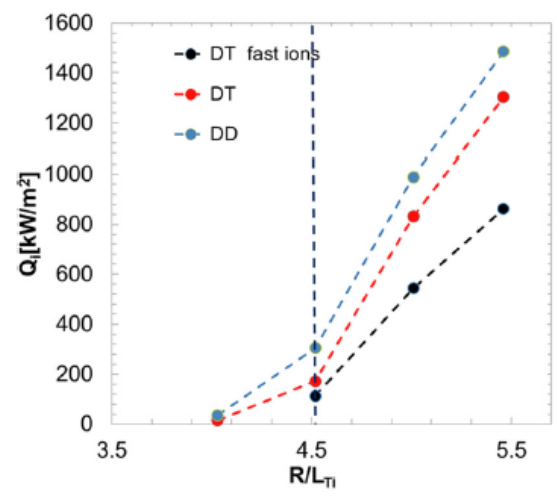

Figure 12. GENE non-linear simulations of an ITER advanced scenario plasma at $\rho_{\text {tor }}=0.33$, showing the stabilizing effect of fast ions. The dashed line corresponds to nominal values. From [71].

\subsection{The effect of isotope mass on transport}

The dependence of transport on the isotope mass of the main ion species has been a longstanding open issue in fusion research, and of high ITER relevance, due to operations foreseen using all 3 Hydrogen isotopes. The GB scaling discussed in Sect. 2 yields a dependence of $\chi$ on $\sqrt{A}$ with $\mathrm{A}$ the mass number, leading to a confinement time $\sim 1 / \sqrt{A}$, i.e. getting worse from $\mathrm{H}$ to $\mathrm{D}$ to $\mathrm{T}$ plasmas. Instead, the opposite trend has been observed experimentally, as shown e.g. by the positive mass exponent of the IPB98(y,2) scaling law [53], which is good for a reactor, but missed for long a proper theoretical explanation. The apparent contradiction has been recently solved by dedicated experiments on JET and AUG and detailed theoretical studies, which are able to model the observed anti-gyroBohm dependence.

First of all, we note that in H-mode the anti-gyroBohm dependence of confinement comes in large part from the pedestal, which is higher in D than in H plasmas, for same plasma parameters, power and fuelling (Figure 13a) [72, 73]. The reasons for this are still under investigation and are outside the scope of this paper. However, even when better matching the edge, such as in L-modes $[59,74]$ or in a recent AUG experiment by matching $\mathrm{H}$-mode pedestal by changing triangularity [75], an anti-gyroBohm or less than gyro-Bohm scaling of the core transport with mass can be observed, depending on plasma conditions [59, 72, 74-78]. An example for a JET L-mode experiment is shown in Figure 13b. This core dependence on mass is the object of this section.
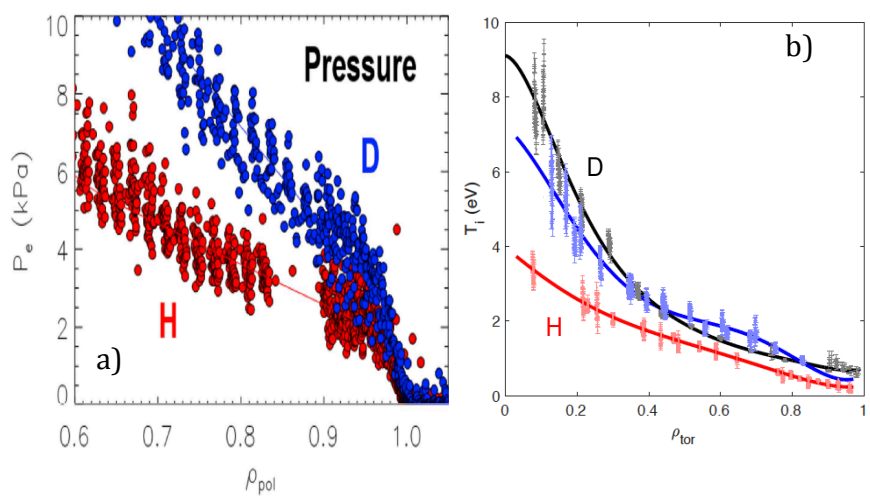

Figure 13. a) Pedestal pressure profiles of JET H-mode plasmas in $\mathrm{D}$ and $\mathrm{H}$ with same heating and fuelling. From [72]. b) $\mathrm{T}_{\mathrm{i}}$ profiles for JET L-mode plasmas in Hydrogen (red) and Deuterium (blue, black) with similar parameters and power levels. From [59].

Non-linear gyro-kinetic investigation has in fact shown that a gyro-Bohm behavior is predicted in a local simulation only for ES fluctuations, adiabatic electrons, no collisions and no background flows. When any of these effects is added, it brings an anti-gyroBohm contribution, so that when all effects are included one can observe a net anti-gyroBohm scaling of the turbulent transport, in agreement with experiments. This is shown in Figure 14 for a case with JET Hydrogen Lmode parameters [76], and similar results for a JET H-mode are reported in $[77,78]$. In addition to these intrinsic turbulence effects, a number of external agents can systematically differ in discharges with different isotopes, e.g. the heating mix, the fast ion population, the particle and momentum sources, collisional equipartition, in a way that leads to antigyroBohm scaling of local core transport. A clear example of this is reported in [59] and shown in Figure 15. An ion heat flux scan like the one shown in Figure 10a has been performed in JET H and D L-mode plasmas with otherwise similar main parameters and heating power, at two power levels. Whilst at low power the data are consistent with gyro-Bohm scaling, at high power deuterium plasmas show much lower ion stiffness than hydrogen plasmas, i.e. an anti-gyroBohm dependence in mass. Two discharges at high power have been modeled with GENE as shown in Figure 15. In these shots the fast ion stabilization is very important, as in the case of Figure 11a. However, due to the NBI system configuration in $\mathrm{H}$ and $\mathrm{D}$, and to the lower ${ }^{3} \mathrm{He}$ concentration required in $\mathrm{H}$ by ICRH minority scheme, the fast ion population is much weaker in the hydrogen shot, inducing less stabilization, and generating the apparent anti-gyroBohm scaling. In conclusion, from the cases of $\mathrm{H}$ vs $\mathrm{D}$ pairs of discharges analyzed in detail with gyro-kinetic simulations so far, we can conclude that when all effects are taken into account, the simulations 
reproduce the experiment, including the anti-gyroBohm dependence when observed.

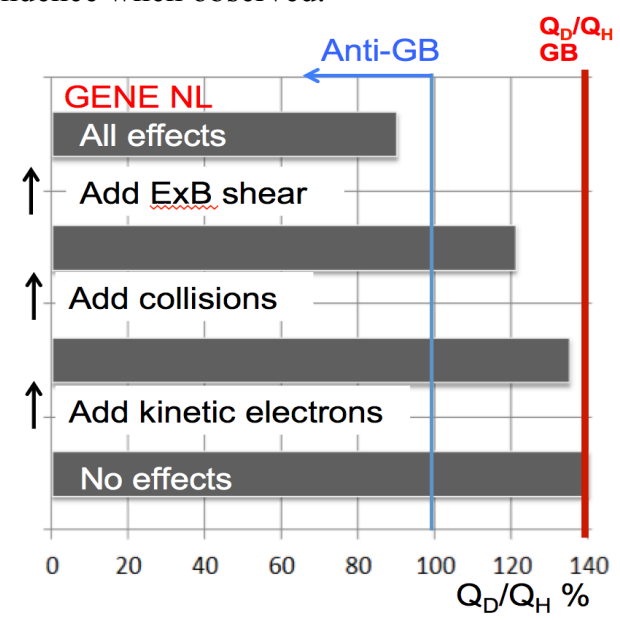

Figure 14. GENE non-linear simulations using JET Hydrogen Lmode plasma parameters, swapping mass from $\mathrm{H}$ to $\mathrm{D}$ and adding various effects that overcome the original gyro-Bohm dependence. From [76].

On the side of quasi-linear modeling in different isotopes instead the validation process is far from being concluded. In general attempts to reproduce an observed anti-gyroBohm trend between $\mathrm{H}$ and $\mathrm{D}$ plasmas have not been successful, as the models yield a gyro-Bohm mass dependence which at most is wiped out by stiffness resulting in no mass scaling, but not in an inverse mass dependence [59, 76, 78]. One example is shown in Figure 15, where at variance with gyrokinetic simulations, which account for the anti-gyroBohm trend via fast ion stabilization, TGLF SAT1, missing such stabilization, yields basically the same prediction (in normalized units) for $\mathrm{H}$ and D plasmas [59]. In terms of profiles, this leads typically to an overestimate of Hydrogen temperature profiles [59, 76], particularly in presence of significant ion heating. This is expected to be cured when a proper modeling of fast ion effects will be included in quasi-linear models, but a thorough validation also with respect to other mechanisms inducing anti-gyroBohm effects is still ongoing, including model validation in $\mathrm{T}$ or $\mathrm{D}-\mathrm{T}$ plasmas.

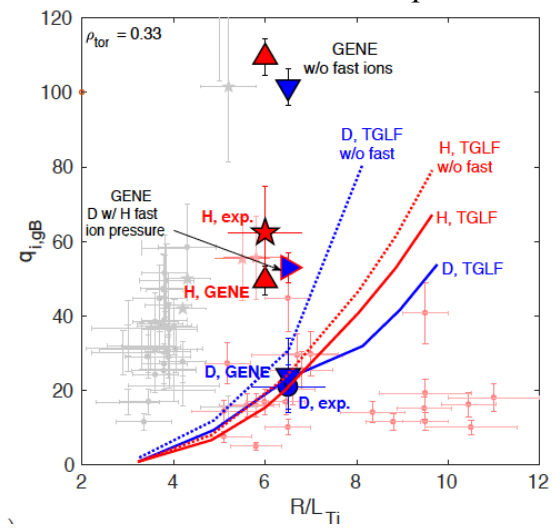

Figure 15. Gyro-Bohm normalized ion heat flux vs $\mathrm{R} / \mathrm{L}_{\mathrm{Ti}}$ for $\mathrm{H}$ (red) and D (blue) JET L-mode pair at high power. Triangles are GENE non-linear simulations without/with fast ions, and lines are TGLF SAT1 simulations without/with fast ion dilution. Also shown is the GENE simulation of the D shot but using the fast ion pressure of the H shot. From [59]. The pale points in background show the whole data-set (grey for low power, red for high power), which can be examined in more detail in [59].

\subsection{The impact of electron scale turbulence and multi-scale interactions}

Electron heat flux scan or heat wave propagation experiments in C-MOD [79], JET [80], DIII-D [81], TCV [82] and AUG [64] have shown that, in conditions of mixed ion and electron heating $\left(\mathrm{T}_{\mathrm{e}} / \mathrm{T}_{\mathrm{i}} \leq 1\right)$ and with high $\mathrm{R} / \mathrm{L}_{\mathrm{Te}}$ drive, the electron heat flux is often too high to be accounted for by ion-scale ITG/TEM instabilities and the electron stiffness is higher than can be justified by TEMs alone. An example for a JET L-mode plasma is shown in Figure 16. In addition, the experimental electron threshold in JET has been found to scale as $\mathrm{Z}_{\text {eff }} \mathrm{T}_{\mathrm{e}} / \mathrm{T}_{\mathrm{i}}$ [80], which is a signature of the ETG threshold [83]. These experimental indications of a possible role of electron scale instabilities require non-linear gyro-kinetic simulations covering both ion and electron scales, to account for their complex coupling. These are computationally very heavy and only few have been performed so far with realistic mass ratio.

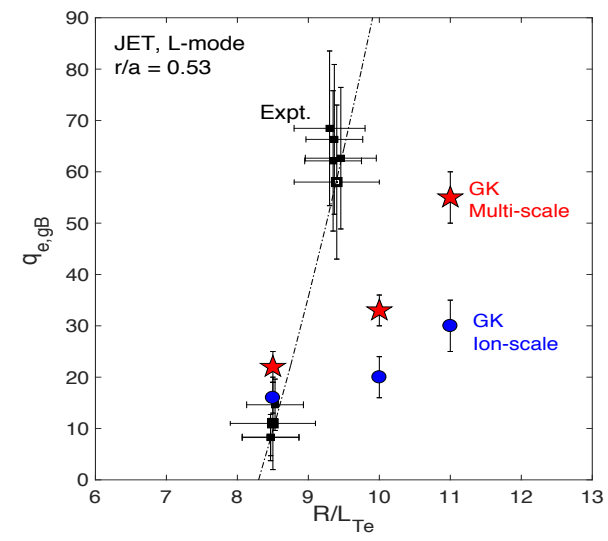

Figure 16. Gyro-Bohm normalized experimental electron heat flux scan (black squares) compared to GENE ion-scale (blue circles) and multi-scale (red stars) non-linear simulations for JET L-mode plasmas. From [80].
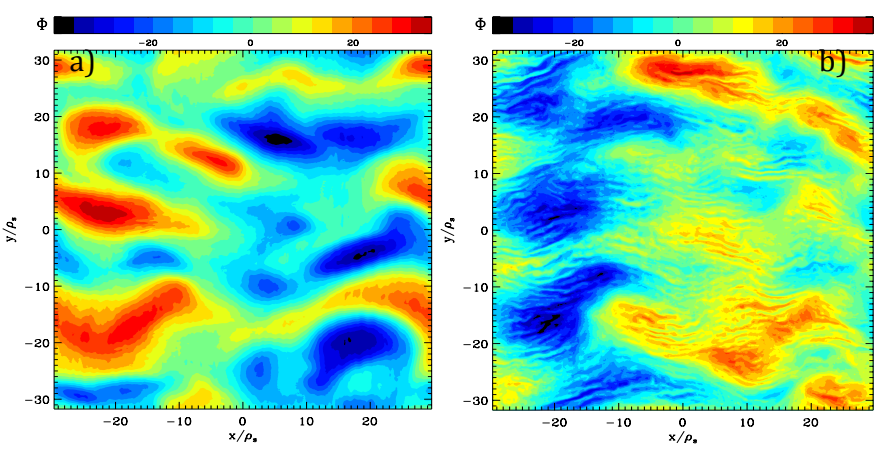

Figure 17. Contour plots of ES potential fluctuations from GENE non-linear multi-scale simulations of the JET experiment of Figure 16 , for $\mathrm{R} / \mathrm{L}_{\mathrm{Te}}=8.5$ (a) and for $\mathrm{R} / \mathrm{L}_{\mathrm{Te}}=11$ (b). From [80].

Figure 17 shows the contour plots of ES potential fluctuations for multi-scale simulations of the JET experiment of Figure 16 , for two values of $\mathrm{R} / \mathrm{L}_{\mathrm{Te}}$ [80]. The low $\mathrm{R} / \mathrm{L}_{\mathrm{Te}}$ case shows turbulence structures only on the ion scales, whilst the high $\mathrm{R} / \mathrm{L}_{\mathrm{Te}}$ case shows the formation of radially elongated structures known as ETG "streamers", carrying significant electron heat flux. In this case an important increase of electron 
heat flux was observed in the multi-scale compared to ionscale simulation, although not fully matching the experimental fluxes (Figure 16). A sensitivity study to look for a better match within the experimental uncertainties of the input parameters is not feasible for this type of heavy simulations.

A C-MOD multi-scale simulation using GYRO is shown in Figure 18, and compared to the ion scale simulation [79]. Here the complex bi-directional interaction between low and high $\mathrm{k}$ portions of the spectrum is clearly documented. For increasing ion drive, the high $\mathrm{k}$ fluctuations are damped by the increasing ion scale zonal flows, whilst near marginality the ETGs are free to develop and can even increase by inverse cascade the low $\mathrm{k}$ flux with respect to a pure ion scale simulation. The figure shows that only the multi-scale simulation can match the experimental ion and electron heat flux levels, in the parameter region close to marginality, with a strong ETG component. As discussed in [79], also the electron stiffness is matched by the multi-scale simulation but not by the ion-scale one. It is worth mentioning that, whilst the multi-scale simulations in $[79,80]$ are ES, an EM multi-scale simulation reported in [84] shows that EM stabilization of ITG by finite beta enhances the importance of cross-scale interactions with ETG, consistently with the dynamics discussed above.
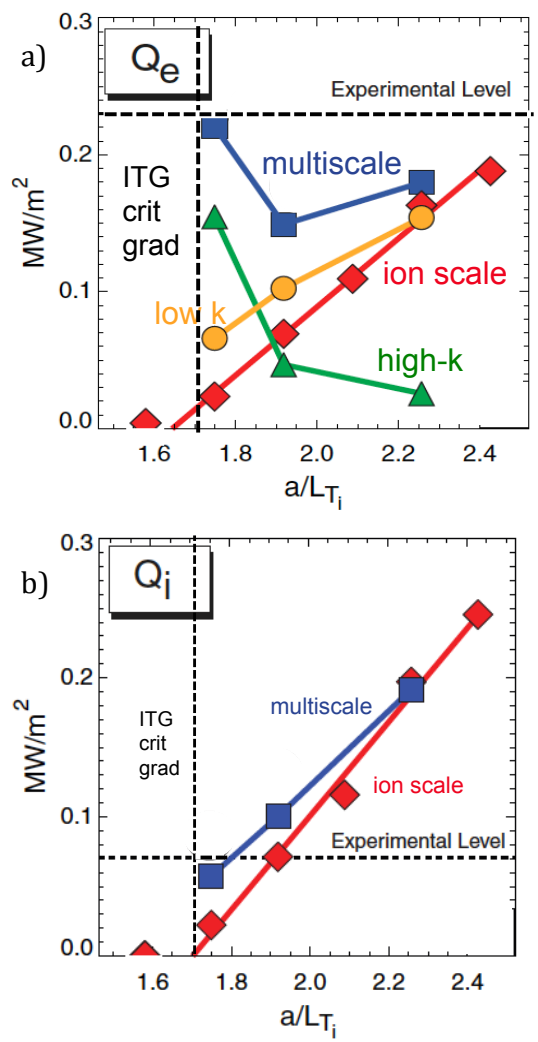

Figure 18. Electron (a) and ion (b) heat flux vs a/ $\mathrm{L}_{\mathrm{Ti}}$ from GYRO multi-scale simulations of a C-MOD experiment $(\mathrm{a} / \mathrm{R}=0.31)$, compared to the ion-scale results and to the experimental levels. The separate contributions to $\mathrm{q}_{\mathrm{e}}$ of low and high $\mathrm{k}$ spectral ranges are indicated. From [79].

Given the indications both from experiment and theory that ETGs can significantly contribute to electron heat transport in some conditions, it is important to have quasi-linear models that reproduce the multi-scale physics, for reliable scenario simulations. This is even more important in view of ITER, where the possible role of ETGs has in fact a potentially more dangerous impact than in most present machines. The latter have significant positive-ion NBI and ICRH heating, which provide via ExB and fast ion stabilization a low ion heat transport, and high ion heating to peak $T_{i}$ irrespective of $T_{e}$ being kept at low threshold by strong ETGs at $\mathrm{T}_{\mathrm{e}} / \mathrm{T}_{\mathrm{i}} \leq 1$. Instead ITER will have dominant electron heating by negativeion NBI, ECH and $\alpha$-particles, so that ions are mainly heated by collisional electron-ion exchange and $\mathrm{T}_{\mathrm{i}}$ cannot exceed $\mathrm{T}_{\mathrm{e}}$. In these conditions, a limitation to $T_{e}$ peaking by ETGs reflects into a limitation on $\mathrm{T}_{\mathrm{i}}$ peaking and fusion power. On the other hand, the higher $\mathrm{T}_{\mathrm{e}} / \mathrm{T}_{\mathrm{i}}(\sim 1)$ itself limits the ETG impact increasing their threshold. In other words, whilst in present ion heated devices ETGs are strong but not deleterious for fusion performance, in electron heated future devices the ETGs are likely not strong, but potentially more deleterious for fusion performance. Therefore an accurate evaluation of the possible role of ETGs in different ITER scenarios is needed, which requires properly validated quasi-linear models including multi-scale physics.

Both TGLF and QuaLiKiz have already been updated to account for ETGs and multi-scale physics, using a linear criterion worked out from the C-MOD multi-scale non-linear simulations, which predicts significant ETG flux when the peak of the $\gamma / \mathrm{k}_{\mathrm{y}}$ spectrum at high $\mathrm{k}_{\mathrm{y}}$ exceeds the peak of the $\gamma / \mathrm{k}_{\mathrm{y}}$ spectrum at low $\mathrm{k}_{\mathrm{y}}[31]$ :

$\left(\frac{\gamma}{k_{y}}\right)^{e, \max }>\left(\frac{\gamma}{k_{y}}\right)^{i, \max }$.

This theoretical criterion has recently received a supportive indication in an electron heat flux scan and modulation experiment in AUG in plasmas with NBI+ECH [64]. In Figure 19a we see GKW linear simulations of such experiment at $\rho_{\text {tor }}=0.5$. We see how for increasing $\mathrm{R} / \mathrm{L}_{\mathrm{Te}}$ the high $\mathrm{k}_{\mathrm{y}}$ peak of $\gamma / \mathrm{k}_{\mathrm{y}}$ increases in proportion to the low $\mathrm{k}_{\mathrm{y}}$ peak of $\gamma / \mathrm{k}_{\mathrm{y}}$. The $\mathrm{R} / \mathrm{L}_{\mathrm{Te}} \sim 6$ value for which GKW indicates the cross-over coincides with the value for which both the heat flux scan and the modulation see an increase in electron stiffness, as shown in Figure $19 \mathrm{~b}$ by the rapidly increasing incremental diffusivity (from heat pulse propagation) vs power balance diffusivity. This supports the appearance of a significant contribution to $\mathrm{q}_{\mathrm{e}}$ by stiff ETGs when the linear criterion is satisfied.

In QuaLiKiz ETGs are switched on when such linear criterion is satisfied, and their contribution to $\mathrm{q}_{\mathrm{e}}$ has been calibrated on non-linear single electron scale GENE simulations of the JET L-mode experiment of Figure 16, as described in [35]. TGLF in the SAT1 version features a saturation model based on zonal flow mixing, which becomes predominant at high $\mathrm{k}_{\mathrm{y}}$ with respect to the zonal flow shearing paradigm used for low $\mathrm{k}_{\mathrm{y}}$ turbulence saturation [31]. The SAT1 model then includes coupling of zonal $\mathrm{k}_{\mathrm{y}}=0$ fluctuations to all $\mathrm{k}_{\mathrm{y}}$-scales and finite $\mathrm{k}_{\mathrm{y}}$ inter-mixing. The model was fit to the GYRO multi-scale simulations for C-MOD shown in Figure 18 and reproduces the transition from the ETG streamer regime to suppressed ETG turbulence as $a / \mathrm{L}_{\mathrm{Ti}}$ is increased. This is presently the most refined reduced model accounting for multi-scale interactions. Both models are under validation against results from the various devices mentioned at the beginning of this sec- 
tion, and yielded significant ETG flux in C-MOD, DIII-D, JET, AUG plasmas with mixed ion and electron heating.
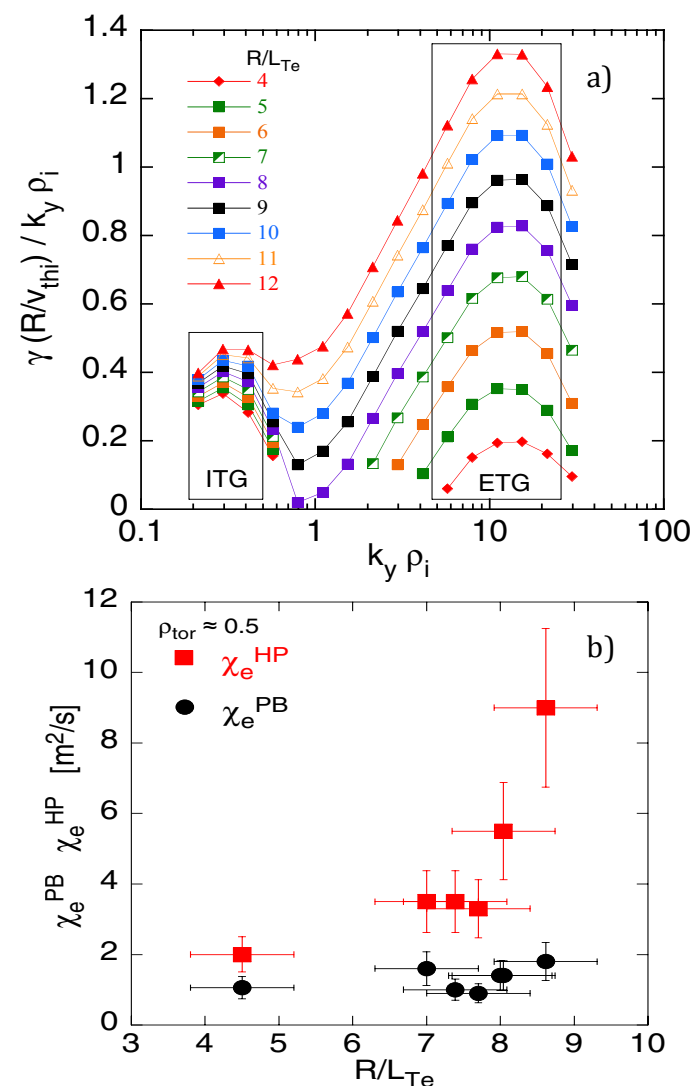

Figure 19. a) Linear $\gamma / \mathrm{k}_{\mathrm{y}}$ spectra at $\rho_{\text {tor }}=0.5$ calculated by GKW at different $\mathrm{R} / \mathrm{L}_{\mathrm{Te}}$ for an AUG electron heat flux scan with $\mathrm{NBI}+\mathrm{ECH}$ power. For $\mathrm{R} / \mathrm{L}_{\mathrm{Te}}>6$ the electron-scale maximum $\gamma / \mathrm{k}_{\mathrm{y}}$ exceeds the ion scale one. b) Power balance and incremental diffusivity (from heat pulse propagation) $v \mathrm{~s} / \mathrm{L}_{\mathrm{Te}}$ measured in an $\mathrm{AUG}$ electron heat flux scan with NBI+ECH power. From [64].

As discussed above, it is then important to evaluate the ETG contribution in the ITER baseline scenario simulation using TGLF SAT1 shown in Figure 8. The spectral distribution of $\mathrm{q}_{\mathrm{e}}$ for such simulation is shown in Figure 20 for $\rho_{\text {tor }}=0.6$. ETGs are clearly destabilized, however the contribution to the total $\mathrm{q}_{\mathrm{e}}$ from the $\mathrm{k}_{\mathrm{y}}>2$ part of the spectrum is $\sim 8 \%$ at the radius shown, and does not exceed $16 \%$ at any radial position, which is reassuring. Still, more ITER regimes have to be evaluated using these models, including plasmas at $1.8 \mathrm{~T}$ with $\mathrm{ECH}$ in the initial phase of operations.

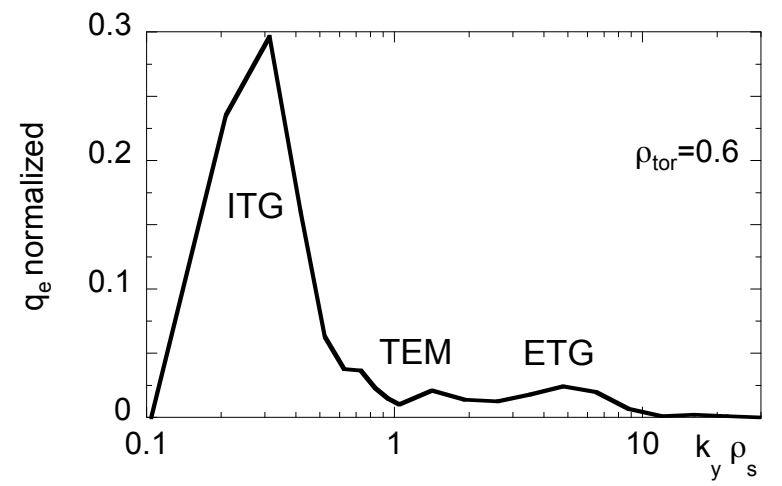

Figure 20. Normalized electron heat flux spectrum at $\rho_{\text {tor }}=0.6$ for the ITER simulation with TGLF SAT1 shown in Fig.8.

\section{Conclusions and outlook}

In summary, after two decades of intense work with continuous feedback between experiments and non-linear GK theory, core turbulent transport is found to be adequately described by local $\delta f \mathrm{GK}$ models in most experimental conditions in present devices, as documented by a vast literature of which this paper offers only few examples. Within the local $\delta f$ framework, more work with GK simulations on multi-scale effects for the cases when such effects are important is still required. Attempts to make profile simulations using local $\delta f$ non-linear GK codes with exascale computing will be extremely interesting, as well as attempts to train neural networks on databases of linear and non-linear GK simulations. With increasing computing power, interesting future work is to test the local of assumptions against even more physics comprehensive global full-f codes, which to date are not systematically validated against experiments when including critical physics like kinetic electrons and EM-effects $[85,86]$. Also GK simulations of the L-mode edge or H-mode pedestal will be an important development, starting from recent attempts in [87-89], and should also lead to more comprehensive reduced models for pedestal transport, beyond the ideal MHD paradigm which is presently mostly adopted in integrated modellling of plasma scenarios [90]. Finally, specific burning plasma physics, such as interaction of meso- and micro-scales [91], will have to be addressed by proper theoretical developments and carefully validated against experiments that are presently not available.

Granted that these developments will be precious, still first principle modeling of tokamak scenarios is already now routine using core turbulence "reduced" quasi-linear models, and reliable predictions of baseline scenarios in present and future machines are available. Continuous improvement of quasilinear models is ongoing, to enrich their physics content and expand their range of validity. Some recently discovered physics mechanisms, such as non-linear EM stabilization, fast ion effects or multi-scale interactions, particularly important for advanced scenarios, have to be properly inserted and/or validated. Validation in different gases or gas mixtures other than the mostly used deuterium will also be required to finally achieve versatile predictive tools for ITER operations. Finally, fast neural network surrogate quasi-linear models are under development for scenario optimization and controloriented applications. This development leverages the enormous recent advances in machine learning techniques, and is based on a regression of quasi-linear transport models using training sets derived from targeted large-scale generation of model input-output mappings [92-96]. These faster versions will allow more large-scale validation and systematic scenario optimization, will facilitate core-pedestal coupled simulations towards full-device physics-based self-consistent scenario predictions, and will contribute to control-oriented applications.

\section{Acknowledgments}

Part of this work has been carried out within the framework of the EUROfusion Consortium and has received funding from the Euratom research and training programme 2014- 
2018 and 2019-2020 under grant agreement No 633053. The views and opinions expressed herein do not necessarily reflect those of the European Commission. This work was also conducted under the auspices of the ITPA Topical Group on Transport \& Confinement.

\section{References}

[1] Freidberg J P 2007 Plasma Physics and Fusion Energy Cambridge University Press

[2] Horton W 1999 Rev. Mod. Physics 71735

[3] Garbet X 2001 Plasma Phys. Control. Fusion 43 A251

[4] Candy J \& Waltz R E 2003 J. Comput . Phys. 186545

[5] courtesy J. Candy and R. Waltz, General Atomics

[6] Garbet X et al 2004 Plasma Phys. Control. Fusion 46 B557

[7] Waltz R E et al 1990 Phys.Rev.Lett 652390

[8] Ryter F et al 2003 Nucl. Fusion 431396

[9] Mantica P \& Ryter F 2006 C. R. Physique 7634

[10] White A E et al 2019 J. Plasma Phys. 85925850102

[11] Brizard A J and Hahm T S 2007 Rev Mod Phys. 79421

[12] Garbet X et al 2010 Nucl. Fusion 50043002

[13] Jenko F et al 2000 Phys Plasmas 71904

[14] Peeters A et al 2009 Comp.Phys.Comm. 1802650

[15] 2017 Plasma Phys. Control. Fusion 59 Special issue on comparing GK simulations to experiments

[16] Creely A J et al 2018 Nucl. Fusion 58126001

[17] Bravenec R et al 2016 Plasma Phys. Control. Fusion 58125018

[18] Rhodes T et al 2011 Nucl. Fusion 51063022

[19] Casati A et al 2009 Phys.Rev.Lett 102165005

[20] Freethy S J et al 2018 Phys. Plasmas 25055903

[21] Holland C et al 2011 Phys. Plasmas 18056113

[22] Goerler T et al 2016 Fusion Science and Techn. 69537

[23] Angioni C et al 2009 Plasma Phys. Control. Fusion 51124017

[24] Angioni C et al 2012 Nucl. Fusion 52114003

[25] Howard N T et al 2012 Nucl. Fusion 52063002

[26] Angioni C et al 2014 Nucl. Fusion 54083028

[27] Manas P et al 2019 Nucl. Fusion 59056014

[28] Peeters A G et al 2011 Nucl. Fusion 51094027

[29] Mariani A et al 2018 Phys. Plasmas 25012313

[30] Staebler G M et al 2007 Phys. Plasmas 14055909

[31] Staebler G M et al 2016 Phys. Plasmas 23062518

[32] Bourdelle C et al 2007 Phys. Plasmas 14112501

[33] Bourdelle C et al 2016 Plasma Phys. Control. Fusion 58 014036

[34] Citrin J et al 2012 Phys. Plasmas 19062305

[35] Citrin J et al 2017 Plasma Phys. Control. Fusion 59124005

[36] Jenko F et al 2005 Plasma Phys. Control. Fusion 47 B195

[37] Lin Z et al 2007 Phys.Rev.Lett L 99, 265003

[38] Kinsey J et al 2011 Nucl. Fusion 51083001

[39] Told D et al 2013 Phys. Plasmas 20122312

[40] Ho A et al 2019 Nucl. Fusion 59056007

[41] Kinsey J E et al 2010 Phys. Plasmas 17122315

[42] Rodriguez-Fernandez P 2018 Fusion Science and Technology 7465

[43] Linder O et al 2019 Nucl. Fusion 59016003

[44] Bonanomi N et al 2018 Nucl.Fusion 58026028

[45] Houlberg W A et al 1997 Phys. Plasmas 43230

[46] Belli E A and Candy J 2012 Plasma Phys. Control. Fusion

54015015

[47] Grierson B A et al 2018 Phys. Plasmas 25022509

[48] Candy J et al 2009 Phys.Plasmas 16060704
[49] Romanelli M. et al 2014 Plasma and Fusion Research 9 3403023

[50] Ho A et al 2019 Nucl. Fusion 59056007

[51] Koechl $\mathrm{F}$ et al 2018 Proc. $27^{\text {th }}$ IAEA FEC, IAEA CN-258

[52] Snyder P B et al 2011 Nucl. Fusion 51103016

[53] ITER Physics Basis: Chapter 21999 Nucl. Fusion 392175

[54] Loarte A et al 2018 Proc. $27^{\text {th }}$ IAEA FEC, IAEA-CN-258/482

[55] ITER organization 2018 ITER Research Plan ITR-18-003

[56] Baiocchi B et al 2015 Plasma Phys. Control. Fusion 57035003

[57] Mantica P 2016 invited talk at $58^{\text {th }}$ APS DPP meeting, S Jose, CA

[58] Mantica $P$ et al 2018 poster at $23^{\text {rd }}$ EU-US Transport Task Force Meeting, Sevilla, ES

[59] Bonanomi N et al 2019 Nucl. Fusion 59096030

[60] Casson F et al 2019 "Predictive multi-channel flux-driven modeling to optimize ICRH tungsten control and fusion performance in JET", submitted to Nucl. Fusion

[61] Mantica P et al 2009 Phys.Rev.Lett 102175002

[62] Mantica P et al 2011 Phys.Rev.Lett 107135004

[63] de Oliveira-Lopes F N et al $201744^{\text {th }}$ EPS Conf. on Plasma Phys., Belfast, Europhysics Conference Abstracts 41F 610

[64] Ryter F et al 2019 Nucl. Fusion 59096052

[65] Citrin J et al 2013 Phys.Rev.Lett 111155001

[66] Di Siena A et al 2018 Nucl. Fusion 58054002

[67] Di Siena A et al 2019 submitted to Nucl Fusion

[68] Whelan G G et al 2018 Phys.Rev.Lett 120175002

[69] Garcia J et al 2015 Nucl. Fusion 55053007

[70] Doerk H et al 2018 Nucl. Fusion 58016044

[71] Garcia J et al 2018 Phys. Plasmas 25055902

[72] Maggi C et al 2018 Plasma Phys. Control. Fusion 60014045

[73] Laggner F et al 2017 Phys. Plasmas 2456105

[74] Schneider P et al 2017 Nucl. Fusion 57066003

[75] Schneider P et al $201924^{\text {th }}$ Joint US-EU Transport Task Force Meeting, Austin, USA

[76] Garcia J et al 2019 Nucl. Fusion 59086047

[77] Oberparlaiter $\mathrm{M}$ et al poster at $23^{\text {rd }}$ EU-US Transport Task Force Meeting, Sevilla, ES

[78] Weisen $\mathrm{H}$ et al 2018 EX/P1-4, IAEA-CN-258 FEC

[79] Howard N et al 2016 Nucl. Fusion 56014004

[80] Bonanomi N et al 2018 Nucl. Fusion 58124003

[81] Smith S P et al 2015 Nucl. Fusion 55083011

[82] Mariani A et al 2019 Nucl. Fusion 59126017

[83] Jenko F et al 2001 Phys. Plasmas 84096

[84] S. Maeyama S et al 2015 Phys. Rev. Lett. 114255002

[85] Idomura Y 2008 Computer Physics Communications 179391

[86] Grandgirard V et al 2007 Plasma Phys. Control. Fu-

sion $49 \mathrm{~B} 173$

[87] Bonanomi N et al 2019 Nucl. Fusion 59126025

[88] Chang C S et al 2017 Phys.Rev.Lett 118175001

[89] Hatch D R et al 2017 Nucl. Fusion 57036020

[90] Snyder P B et al 2009 Nucl. Fusion 49085035

[91] Zonca F et al 2015 Plasma Physics and Controlled Fusion 57 014024

[92] Citrin J et al 2015 Nucl. Fusion 55092001

[93] Meneghini O et al 2017 Nucl. Fusion 57086034

[94] Felici F et al 2018 Nucl. Fusion 58096006

[95] Meneghini O et al "Self-consistent neural-network accelerated modelling of fusion plasmas compatible with ITER IMAS" to be submitted to Nucl. Fusion

[96] van de Plassche K L et al 2019 "Fast modelling of turbulent transport in fusion plasmas using neural networks" submitted to Phys. Plasmas 